\title{
Bright Gamma-ray Pulsars in the Fermi Era : Results and prospects with ground-based telescopes
}

\section{Thomas Tavernier*}

Astroparticule et Cosmologie, universite Paris 7

E-mail: tavernie@apc.univ-paris7.fr

\begin{abstract}
With more than 140 gamma-ray emitting pulsars detected, the Large Area Telescope (LAT) aboard the Fermi satellite has revolutionized the field of high-energy pulsar astronomy.

While the processes (e.g. curvature radiation, synchrotron, Inverse-Compton emission) thought to be responsible for the pulsed emission at high energies are well known, the details of the acceleration and radiation mechanisms at play are still widely debated through several models.

The analysis of the large data-set of events available from the brightest gamma-ray pulsars, six years after the launch of the Fermi satellite, reveals for these individuals a harder spectrum than previously measured (and expected) and hence increases the chance to detect them with groundbased Cherenkov Telescopes. The detection from ground at the highest energies brings in turn precious information allowing one to constrain severely existing models. The latest results will be presented, and prospects in the field will be discussed.
\end{abstract}

Frontiers of Fundamental Physics 14 - FFP14,

15-18 July 2014

Aix Marseille University (AMU) Saint-Charles Campus, Marseille

\footnotetext{
*Speaker.
} 